\title{
Exact Scaling Solutions in Normal and Brans-Dicke Models of Dark Energy
}

\author{
Olga Arias ${ }^{1}$, Tame Gonzalez ${ }^{2}$, Yoelsy Leyva ${ }^{3}$ and Israel Quiros ${ }^{4}$ \\ Physics Department. Las Villas Central University. \\ Santa Clara 54830. Villa Clara. Cuba
}

\begin{abstract}
A linear relationship between the Hubble expansion parameter and the time derivative of the scalar field is explored in order to derive exact cosmological, attractor-like solutions, both in Einstein's theory and in Brans-Dicke gravity with two fluids: a background fluid of ordinary matter, together with a self-interacting scalar field accounting for the dark energy in the universe. A priori assumptions about the functional form of the self-interaction potential or about the scale factor behavior are not necessary. These are obtained as outputs of the assumed relationship between the Hubble parameter and the time derivative of the scalar field. A parametric class of scaling quintessence models given by a self-interaction potential of a peculiar form: a combination of exponentials with dependence on the barotropic index of the background fluid, arises. Both normal quintessence described by a self-interacting scalar field minimally coupled to gravity and Brans-Dicke quintessence given by a non-minimally coupled scalar field are then analyzed and the relevance of these models for the description of the cosmic evolution are discussed in some detail. The stability of these solutions is also briefly commented.
\end{abstract}




\section{Introduction}

Dark energy or missing energy is one of the contemporary issues the physics community is more interested in due, mainly, to a relatively recent (revolutionary) discovery that our present universe is in a stage of accelerated expansion[1]. This missing component of the material content of the universe is the responsible for the current stage of accelerated expansion and accounts for $2 / 3$ of the total energy content of the universe, determining its destiny[2]. This is a new form of energy with repulsive gravity and possible implications for quantum theory and supersymmetry breaking. Among others, the new cosmology is characterized by the following features[2]: 1) Flat, critical density accelerating universe, 2) early period of inflation, 3) density inhomogeneities produced from quantum fluctuations during inflation, 4) composition of 2/3 dark energy, 1/3 dark matter and 1/200 bright stars, 5) matter content: $(29 \pm 4) \%$ cold dark matter, $(4 \pm 1) \%$ baryons and $\sim 0.3 \%$ neutrinos. Besides, there is evidence (recent observation of SN1997ff at redshift $z=1.7$ ) that the present stage of accelerated expansion was preceded by an early period of decelerated expansion[2]. Any theoretical framework intended to describe our present universe should be compatible with most of these requirements. ${ }^{5}$

A self-interacting, slowly varying scalar field, most often called quintessence, has been meant to account for the dark energy component. In the simplest models of quintessence, minimally coupled scalar fields are considered. However, extended quintessence models have also been considered $[3,4,5]$. In these models the scalar field that accounts for the dark energy component is non-minimally coupled to gravity and, besides, is a part of the gravity sector. Brans-Dicke theory is a prototype for this kind. Motivated by the possibility to have superaccelerated expansion and, correspondingly, superquintessence solutions, this type of theories is now being considered (see, for instance, Ref.[5] and references therein).

A variety of self-interaction potentials for the quintessence field has been studied. Among them, the simplest exponential potential (a single exponential) model is unacceptable because it can not produce the transition from subdominant to dominant energy density[6]. Other combinations of exponential potentials have been also studied $[7,8,9,10,11]$. Combinations of exponentials are interesting alternatives since these arise in more fundamental (particle) contexts: supergravity and superstring[12], where these types of potentials appear after dimensional reduction.

In most cases the occurrence of a self-interaction potential for the scalar field makes difficult to solve analytically the field equations, although some techniques for deriving solutions have been developed. In Ref.[13], for instance, the form of the scale factor is given a priori and, consequently, the self-interaction potential can be found that obeys the field equations. This method has been repeatedly used[14, 15] as well as interesting applications of it[16]. However there are cases when exact solutions can be found once the form of the potential is given $[10,11]$. In other cases some suitable relationship between the self-interaction potential and the scalar field kinetic energy is assumed[17]. The assumption that the scalar field

\footnotetext{
${ }^{5}$ Most part of these features can be considered as observational evidence. Early inflation, on the contrary, is just a theoretical model that is consistent with observations.
} 
energy density scales as an exact power of the scale factor has also been used for deriving solutions [18].

In case we deal with a non-minimally coupled scalar field that accounts for the dark energy, the situation with finding exact solutions is even more complicated. It seems that numerical integration is the "simplest" way to treat this problem. In Ref. [5], for instance, numerical solutions are explored in extended quintessence models.

In this paper we explore a linear relationship between the Hubble expansion parameter and the time derivative of the scalar field to derive exact cosmological scaling solutions ${ }^{6}$ both in Einstein's gravity and in Brans-Dicke theory with two fluids: a barotropic perfect fluid of ordinary matter, together with a self-interacting scalar field fluid accounting for the dark energy in the universe.

A similar (but more general) relationship $\dot{\phi} \propto H+\alpha L(\phi)$ has been used in Ref. [20] to find general solutions in $N+1$-dimensional theories of gravity with a self- interacting scalar field and also to derive general solutions when one deals with two scalars: a self-interacting scalar field with exponential potential plus a free scalar field. ${ }^{7}$

We show that the assumed linear relationship between the Hubble parameter and the time derivative of the scalar field is suggested by an implicit symmetry of the field equations and it can lead always to attractor scaling solutions. In this regard, we point out that it is not necessary to make any a priori assumptions about the functional form of the self-interaction potential or about the scale factor behavior. These are obtained as outputs of the assumed relationship once one integrates the field equations explicitly. As a result a peculiar class of potentials arise: a combination of exponentials with some dependence on the barotropic index of the ordinary matter fluid. This kind of potential is of interest for models of dark energy since, as it has been pointed out in Ref. [12], a single exponential potential can lead to one of the following scaling attractor solutions: 1) a late time attractor where the scalar field mimics the evolution of the background barotropic fluid, and 2) the late time attractor is the scalar field dominated solution. In consequence, a combination of exponentials should allow for a scenario in which the universe evolves through a radiation-matter regime (attractor 1 ) and at some recent epoch evolves into the scalar field dominated regime (attractor 2).

In this paper we will be concerned with flat Friedmann-Robertson-Walker (FRW) cosmologies with the line element given by:

$$
d s^{2}=-d t^{2}+a(t)^{2} \delta_{i k} d x^{i} d x^{k}
$$

where the indexes $i, k=1,2,3$ and $a(t)$ is the scale factor. We use the system of units in which $8 \pi G=c=1$.

The paper has been organized in the following way. In section 2 we explain the details of the method by applying it to derive solutions in Einstein's theory with two fluids: a background fluid of ordinary matter and a fluid of a self-interacting scalar field that accounts

\footnotetext{
${ }^{6}$ By scaling we mean cosmological models where the dimensionless density parameter for each component in the energy content is a constant: $\Omega_{i} \equiv \rho_{i} / \rho_{\text {crit }}=$ constant [19].

${ }^{7} \mathrm{~A}$ method where a linear relationship between the field variables and/or their derivatives is assumed has been also used in [21] to derive 4d Poincare invariant solutions in thick brane contexts.
} 
for the dark energy component in the universe. We study also, the resulting parametric class of scaling solutions (including the space of parameters) and we briefly comment on their stability. In particular, we make clear the point that the assumed linear relationship between the Hubble expansion parameter and the time derivative of the scalar field, acts just like a selection principle that preserves only the (relevant) attractor-like solutions. Section 3 is devoted to the derivation of a parametric class of scaling solutions in Brans-Dicke gravity with a background fluid and a self-interacting Brans-Dicke (BD) field, by assuming the same linear relationship between the Hubble expansion parameter and the time derivative of the BD field. We apply the conformal technique to simplify the mathematical handling of the problem. The stability of the solutions found is also commented in this section, while their relevance for the description of the cosmic evolution will be discussed in some detail in section 4. Some conclusions are given in the final section 5 .

\section{The method}

In this section we explain the details of the method we use for deriving solutions in gravity coupled to self-interacting scalars. We will derive solutions in Einstein's gravity with two fluids: a background barotropic fluid of ordinary matter and a self-interacting scalar field fluid. The field equations are the following:

$$
\begin{gathered}
3 H^{2}=\rho_{m}+\frac{1}{2} \dot{\phi}^{2}+V, \\
2 \dot{H}+3 H^{2}=(1-\gamma) \rho_{m}-\frac{1}{2} \dot{\phi}^{2}+V, \\
\ddot{\phi}+3 H \dot{\phi}=-V^{\prime},
\end{gathered}
$$

where $V=V(\phi)$ is the self-interaction potential, $\gamma$ is the barotropic index of the background fluid and $H=\frac{\dot{a}}{a}$ is the Hubble expansion parameter. The dot accounts for derivative in respect to the comoving time $t$ meanwhile the comma denotes derivative in respect to the scalar field $\phi$. The energy density of the ordinary matter (cold dark matter plus baryons and/or radiation) is related with the scale factor through $\rho_{m}=\rho_{0, \gamma} a^{-3 \gamma}$, where $\rho_{0, \gamma}$ is an integration constant coming from integrating the conservation equation. Let us combine equations (2) and (3) to obtain

$$
\dot{H}+3 H^{2}=\frac{2-\gamma}{2} \rho_{m}+V
$$

An implicit symmetry of the left hand side (LHS) of equations (4) and (5)is evident under the change $H \rightarrow k \dot{\phi}$. I. e., if one assumes a linear relationship between the Hubble expansion parameter and the time derivative of the scalar field;

$$
H=k \dot{\phi}, \Rightarrow a=e^{k \phi}
$$


where $k$ is a constant parameter, the LHS of equations (4) and (5) coincide up to the factor $k$. We obtain a differential equation for determining the functional form of the potential $V$ :

$$
V^{\prime}+\frac{1}{k} V=\frac{\gamma-2}{2 k} \rho_{0, \gamma} e^{-3 k \gamma \phi} .
$$

Explicit integration yields the following potential which is a combination of exponentials:

$$
V=\xi_{0} e^{-\phi / k}+\frac{2-\gamma}{6 k^{2} \gamma-2} \rho_{0, \gamma} e^{-3 k \gamma \phi},
$$

where $\xi_{0}$ is an integration constant. This potential is a particular case of the potential $V(\phi)=$ $M^{4}\left(e^{\alpha \phi}+e^{\beta \phi}\right)$ in Ref.[12], when $\alpha=1 / k, \beta=3 k \gamma$, and $\xi_{0}=\frac{2-\gamma}{6 k^{2} \gamma-2} \rho_{0, \gamma}=M^{4}$. It can be given in terms of the scale factor if one considers equation (6): $V=\xi_{0} a^{-1 / k^{2}}+\frac{2-\gamma}{6 k^{2} \gamma-2} \rho_{0, \gamma} a^{-3 \gamma}$.

An interesting feature of this potential is that it depends on the type of ordinary fluid which fills the universe. Otherwise, it depends on the barotropic index $\gamma$ of the matter fluid. If one introduces the following time variable $d t=a^{1 / 2 k^{2}} d \tau$ and by substituting (8) back into Eq. (2), one can rewrite this last equation in the following form:

$$
(\dot{a} / a)^{2}=\frac{2 k^{2} \gamma}{6 k^{2}-2} \rho_{0, \gamma} a^{-3 \gamma+1 / k^{2}}+\frac{2 k^{2} \xi_{0}}{6 k^{2}-1},
$$

where now the dot means derivative in respect to the new time variable $\tau$. This equation can be integrated in quadratures:

$$
\int \frac{a^{3 \gamma / 2-1 / 2 k^{2}-1} d a}{\sqrt{A+B a^{3 \gamma+1 / k^{2}}}}=\tau+\tau_{0},
$$

where $A=\frac{2 k^{2} \gamma}{6 k^{2} \gamma-2} \rho_{0, \gamma}, B=\frac{2 k^{2} \xi_{0}}{6 k^{2}-1}$, and $\tau_{0}$ is an integration constant.

\subsection{The class of solutions}

The integral in the LHS of Eq. (10) can be explicitly taken to yield the following expression for the scale factor as function of the time variable $\tau$;

$$
a(\tau)=a_{0}\left\{\sinh \left[\mu\left(\tau+\tau_{0}\right)\right]\right\}^{2 k^{2} /\left(3 k^{2} \gamma-1\right)},
$$

where $a_{0}=(A / B)^{k^{2} /\left(3 k^{2} \gamma-1\right)}$ and $\mu=\left(3 k^{2} \gamma-1\right) \sqrt{B} / k^{2}$. Then, according to Eq. (6) the scalar field can be easily computed to yield,

$$
\phi(\tau)=\frac{1}{k} \ln a_{0}+\frac{2 k}{3 k^{2} \gamma-1} \ln \sinh \left[\mu\left(\tau+\tau_{0}\right)\right] .
$$

The Hubble expansion parameter can be computed by the knowledge of the scale factor (11):

$$
H(\tau)=\sqrt{\frac{2 k^{2}}{6 k^{2}-1} \xi_{0}} a(\tau)^{-1 / 2 k^{2}} \operatorname{coth}\left[\mu\left(\tau+\tau_{0}\right)\right]
$$


For the purpose of observational testing of the solutions it is useful to look for another magnitudes of astrophysical interest. Among them, the matter density parameter:

$$
\Omega_{m}=\rho_{m} / 3 H^{2}=\frac{3 k^{2} \gamma-1}{3 k^{2} \gamma}\left\{\cosh \left[\mu\left(\tau+\tau_{0}\right)\right]\right\}^{-2},
$$

the scalar field energy density and scalar field density parameter:

$$
\begin{gathered}
\rho_{\phi}=\frac{1}{2} \dot{\phi}^{2}+V=3 H^{2}-\rho_{m}, \\
\Omega_{\phi}=\frac{\rho_{\phi}}{3 H^{2}}=1-\Omega_{m},
\end{gathered}
$$

respectively. The second equation in (15) is just another writing for the field equation (2). The scalar field state parameter can be written in the following form:

$$
\omega_{\phi}=\frac{\frac{1}{2} \dot{\phi}^{2}-V}{\frac{1}{2} \dot{\phi}^{2}+V}=-1+\frac{1}{3 k^{2}\left(1-\Omega_{m}\right)},
$$

meanwhile the deceleration parameter $q=-\left(1+\dot{H} / H^{2}\right)$ :

$$
q=-1+\frac{1}{2 k^{2}}+\frac{3 \gamma}{2} \Omega_{m}
$$

While deriving equations (15)-(17) we have used the field equations (2), (3), (4) and their combinations. Assuming the linear relationship (6) between the Hubble parameter and the time derivative of the scalar field means that we are introducing a new free parameter, however, as it will be shown latter on in subsection 2.B, the space of parameters can be reduced by the combined action of choosing an appropriated normalization and considering some well established observational facts. For purpose of observational testing of the solution it is desirable to have the physical parameters of observational interest written as functions of redshift $z$. This is achieved simply by replacing in the former equations

$$
\Omega_{m}(\tau) \rightarrow \Omega_{m}(z)=\left(\frac{\rho_{0, \gamma}}{3 A}\right) \frac{(1+z)^{2 n}}{(1+z)^{2 n}+B / A},
$$

where $2 n=3 \gamma-1 / k^{2}$. While deriving this expression one has considered $a(z)=a(0) /(z+1)$, and the normalization $a(0)=1$. In terms of the redshift the Hubble expansion parameter can be written as:

$$
H(z)=\sqrt{A} \sqrt{(z+1)^{3 \gamma}+\frac{B}{A}(z+1)^{1 / k^{2}}},
$$

meanwhile, the background energy density $\rho_{m}=\rho_{0, \gamma}(z+1)^{3 \gamma}$. 


\subsection{The space of parameters}

We now investigate the space of parameters $\left(\gamma, \xi_{0}, \rho_{0 \gamma}, k\right)$ parameterizing the solutions (the parameter $\gamma$ can be fixed to be 1 at present and $4 / 3$ in the past). We consider $k^{2}>0$ since otherwise the scalar field would be a complex one. We should differentiate two ranges. For $0<k^{2}<1 / 3 \gamma$ the scale factor evolves periodically:

$$
a(\tau)=\tilde{a}_{0}\left\{\sin \left[\tilde{\omega}\left(\tau+\tau_{0}\right)\right]\right\}^{2 k^{2} /\left(3 k^{2} \gamma-1\right)},
$$

where $\tilde{a}_{0}=(-A / B)^{k^{2} /\left(3 k^{2} \gamma-1\right)}$. However, in this case, for $0 \leq \gamma \leq 2,3 k^{2} \gamma-1<0$ so one should have $\xi_{0}<0$. This means that the self-interaction potential itself is negative, i. e., the scalar field energy density is negative. Besides $q>0$ always and this case is not of observational interest. Therefore we drop this interval in parameter $k$.

For $k^{2}>1 / 3 \gamma$ the equations (11)-(13) are valid in that form. To constrain further the space of parameters we use some observational facts. However, this point is left for further discussion in section 4 . We now determine the constant $A / B$. For this purpose one uses two observational facts: 1 ) Since, at present $\Omega_{m}(0)=1 / 3$, then one obtains from Eq. (18) that:

$$
A+B=\rho_{0, \gamma},
$$

and 2) Since radiation-matter dominated the early stages of the evolution, then one may require that $\Omega_{m}(\infty)=1-\varepsilon$, where the new parameter $\varepsilon<<1$. This small parameter is necessary since a matter-dominated $\left(\Omega_{m}=1\right)$ is not stable [22]. In consequence, from the same Eq.(18), one obtains that:

$$
\rho_{0, \gamma}=3 A(1-\varepsilon) .
$$

Combining the above equalities one gets that: $\frac{B}{A}=2-3 \varepsilon$. In consequence:

$$
\Omega_{m}(z)=(1-\varepsilon) \frac{(z+1)^{2 n}}{(z+1)^{2 n}+2-3 \varepsilon},
$$

and,

$$
H(z)=\sqrt{\rho_{o, \gamma}} \sqrt{\frac{(z+1)^{3 \gamma}+(2-3 \varepsilon)(z+1)^{\frac{1}{k^{2}}}}{3(1-\varepsilon)}} .
$$

One can reduce the space of parameters $\left(\gamma, \varepsilon, \rho_{0, \gamma}, k\right)$ by requiring the following normalization: $H(0)=1$, implying that $\rho_{m}(0)=\rho_{0, \gamma}=1$. We are then left with a three-parameter class of solutions spanned by $(\gamma, \varepsilon, k)$. In section 4 we will further constrain the parameters $\varepsilon$ and $k$ by the observations.

\subsection{Stability of the solutions}

The stability analysis of the model given by Eqs.(2.1)-(2.3) has been performed in Ref.[22] for the class of exponential potentials of the kind: $V=V_{0} e^{-\lambda \phi}(k=8 \pi G=1)$. There are up to 
five fixed points (critical points). Two of them are late-time attractor solutions. One of these is the scalar field dominated solution: $\Omega_{\phi}=1$, with an affective barotropic index $\gamma_{\phi}=\lambda^{2} / 3$. This solution exist for $\lambda^{2}<6$, and is a lite-time attractor in the presence of a background fluid when $\lambda^{2}<3 \gamma[22]$. The other attractor solution occurs for $\lambda^{2}>3 \gamma$ and neither the scalar-field nor the background fluid entirely dominates the evolution.It is a scaling solution where $\Omega_{\phi}=\frac{3 \gamma}{\lambda^{2}}$. At this point we want to discuss on the implications of the linear relationship (2.5) assumed in order to find exact solutions. The procedure for the stability analysis [22] implies writing the evolution equations (2.1)-(2.3) as a plane-autonomous system. For this purpose, one should define new variables:

$$
x \equiv \frac{\dot{\phi}}{\sqrt{6} H}, \quad y \equiv \frac{\sqrt{V}}{\sqrt{3} H} .
$$

Considering (6), the first equation implies $x=\frac{1}{k \sqrt{6}}$. Meanwhile the critical points leading to the former attractor solutions are $x_{1}=\lambda_{1} / \sqrt{6}$ for the scalar dominated solution and $x_{2}=\sqrt{\frac{3}{2}} \gamma / \lambda_{2}$ for the scaling solution [22]. On the other hand, our potential (Eq.(8)) contains a combination of exponentials with exponents $\lambda_{1}=1 / k$ and $\lambda_{2}=3 k \gamma$. We see then, that assuming the linear relationship (6) means dropping of the critical points but the ones that can lead to attractor solutions. Otherwise, relationship (6) could be written in the following equivalent way: $\gamma_{\phi} \Omega_{\phi}=\frac{1}{3 k^{2}}$. Then one is faced with two possibilities: either $\Omega_{\gamma}=1 \Rightarrow \gamma_{\phi}=\frac{1}{3 k^{2}}$ (scalar field dominated solution), or $\gamma_{\phi}=\gamma, \quad \Omega_{\phi}=\frac{1}{3 k^{2} \gamma}=$ const (scaling solution). In this sense one can say that the assumed relationship between $H$ and $\dot{\phi}$ (Eq. (6)) acts just like a selection principle that, among the possible critical points, preserves only the (relevant) attractor-like solutions.

\section{Brans-Dicke quintessence}

Now we use the former method to derive exact solutions in Brans-Dicke gravity with a self-interacting scalar field. As mentioned in the introduction to this paper, this is one in a kind of models of extended quintessence studied before in [3, 4, 5]. In Ref. [5], in particular, numerical solutions in extended models of quintessence (including Brans-Dicke) were explored.

The Brans-Dicke field equations, in the presence of a self-interacting scalar field, are:

$$
\begin{gathered}
3 H^{2}+3 H \dot{\varphi}-\frac{\omega}{2} \dot{\varphi}^{2}=e^{-\varphi}\left(\rho_{m}+V\right), \\
2 \dot{H}+3 H^{2}+\ddot{\varphi}+\dot{\varphi}^{2}+2 H \dot{\varphi}+\frac{\omega}{2} \dot{\varphi}^{2}=e^{-\varphi}\{(1-\gamma)\} \rho_{m}+V, \\
\ddot{\varphi}+\dot{\varphi}^{2}+3 H \dot{\varphi}=\frac{e^{-\varphi}}{2 \omega+3}\left\{(4-3 \gamma) \rho_{m}+4 V-2 V^{\prime}\right\},
\end{gathered}
$$


where we have introduced a new field variable $\varphi$ instead of the original $\phi=e^{\varphi}$. In these equations $\omega$ is the Brans-Dicke coupling parameter, $\gamma$ is the barotropic index of the fluid of ordinary matter, $V$ is the self-interaction potential and $H=\frac{\dot{a}}{a}$ is the Hubble expansion parameter. The dot accounts for derivative in respect to the comoving time $t$ meanwhile the comma denotes derivative in respect to the scalar field $\varphi$. As before, the energy density of the ordinary matter (cold dark matter plus baryons and/or radiation) is related with the scale factor through $\rho_{m}=\rho_{0, \gamma} a^{-3 \gamma}$, where $\rho_{0, \gamma}$ is an integration constant coming from integrating the conservation equation.

For simplicity of the mathematical handling of the problem it is convenient to perform a conformal transformation of the metric: $\bar{g}_{a b}=e^{\psi / \sqrt{\xi}} g_{a b}$, where $\xi=\omega+3 / 2$ and, for convenience, we have rescaled the $\mathrm{BD}$ field $\varphi=\psi / \sqrt{\xi}$. The transformed line element is now: $d \bar{s}^{2}=-d \bar{t}^{2}+\bar{a}^{2} \delta_{i j} d x^{i} d x^{j}$, where the conformal comoving time and scale factor are related with the original (Jordan frame) ones through: $d t=e^{-\psi /(2 \sqrt{\xi})} d \bar{t}$, and $a=e^{-\psi /(2 \sqrt{\xi})} \bar{a}$ respectively. Then we will be faced with the Einstein frame (EF) formulation of BD theory. The relevant field equations are now the following:

$$
\begin{gathered}
3 \bar{H}^{2}=\bar{\rho}_{m}+\bar{\rho}_{\psi}, \\
2 \dot{\bar{H}}+3 \bar{H}^{2}=(1-\gamma) \bar{\rho}_{m}-\bar{P}_{\psi}, \\
\ddot{\psi}+3 \bar{H} \dot{\psi}=\frac{4-3 \gamma}{2 \sqrt{\xi}} \bar{\rho}_{m}-\bar{V}^{\prime},
\end{gathered}
$$

where the EF scalar field energy density $\bar{\rho}_{\psi}=\frac{1}{2} \dot{\psi}^{2}+\bar{V}$ and the corresponding pressure $\bar{P}_{\psi}=\frac{1}{2} \dot{\psi}^{2}-\bar{V}$. Besides, the transformed "conservation" equation is:

$$
\dot{\bar{\rho}}_{m}+\left(3 \gamma \bar{H}+\sqrt{\frac{2}{3}} W \dot{\psi}\right) \bar{\rho}_{m}=0
$$

where the "exchange" function $W=\sqrt{\frac{3}{2}} \frac{4-3 \gamma}{2 \sqrt{\xi}}$ has been introduced as in Ref.[19]. After integration: $\bar{\rho}_{m}=\bar{\rho}_{0, \gamma} \bar{a}^{-3 \gamma} e^{-\sqrt{\frac{2}{3}} W \psi}$. Summing up (29) and (30) yields:

$$
\dot{\bar{H}}+3 \bar{H}^{2}=\frac{2-\gamma}{2} \bar{\rho}_{m}+\bar{V}
$$

As before an implicit symmetry of the LHS of equations (31) and (33) is made explicit by the change $\dot{\psi} \rightarrow \lambda \bar{H}$. In this case Eq.(31) $\rightarrow \lambda$ times Eq.(33). Therefore, if we assume the linear relationship

$$
\dot{\psi}=\lambda \bar{H}, \Rightarrow e^{\psi}=\bar{a}^{\lambda}
$$

then the following differential equation for the self-interaction potential $V$ is obtained: $\bar{V}^{\prime}+$ $\lambda \bar{V}=\left[2 \sqrt{\frac{2}{3}} W-(2-\gamma) \lambda\right] \bar{\rho}_{m} / 2$. Straightforward integration of this equation yields: 


$$
\bar{V}(\psi)=\bar{V}_{0} e^{-\lambda \psi}+\bar{W}_{0} e^{-\delta \psi},
$$

where $\delta=3 \gamma / \lambda+\sqrt{2 / 3} W, \bar{V}_{0}$ is an integration constant and $\bar{W}_{0}=\left[\sqrt{\frac{2}{3}} W-\frac{2-\gamma}{2} \lambda\right] \bar{\rho}_{0, \gamma} /(\lambda-\delta)$. In terms of the scale factor Eq. (35) reads: $\bar{V}(\psi(\bar{a}))=\bar{V}_{0} \bar{a}^{-\lambda^{2}}+\bar{W}_{0} \bar{a}^{-\delta \lambda}$.

This potential belongs to the same class that (8) and shows the interesting feature that it depends on the type of ordinary fluid which fills the universe (it depends on the barotropic index $\gamma$ of the matter fluid). By substituting Eq. (35) back into the Friedmann equation (29) one obtains

$$
\bar{H}^{2}=A \bar{a}^{-\lambda \delta}+B \bar{a}^{-\lambda^{2}}
$$

where $A=\left(\lambda^{2}-6\right) \gamma /\left(\lambda^{2}-\sqrt{2 / 3} W \lambda-3 \gamma\right), B=2 \bar{V}_{0} /\left(6-\lambda^{2}\right)$, and we have considered, besides, that $\bar{\rho}_{m}=\bar{\rho}_{0, \gamma} \bar{a}^{-3 \gamma-\sqrt{\frac{2}{3}} W \lambda}=\bar{\rho}_{0, \gamma} \bar{a}^{-\delta \lambda}$.

To solve (36) we make another change of time variable: $d r=\bar{a}^{-\lambda^{2} / 2} d \bar{t}$ to get Eq. (36) integrated in quadratures:

$$
\int \frac{\bar{a}^{\lambda(\delta-\lambda) / 2-1} d \bar{a}}{\sqrt{\frac{A}{B}+\bar{a}^{\lambda(\delta-\lambda)}}}=\sqrt{B}\left(r+r_{0}\right)
$$

where $r_{0}$ is another integration constant.

\subsection{The class of solutions}

Straightforward integration of Eq. (37) leads to:

$$
\bar{a}(r)^{\lambda(\delta-\lambda) / 2}=\sqrt{A / B} \sinh \left[\mu\left(r+r_{0}\right)\right],
$$

with $\mu=\sqrt{B / \xi} \lambda(\delta-\lambda) / 2$. For the rescaled BD scalar field we obtain:

$$
e^{\psi}=\left\{\sqrt{A / B} \sinh \left[\mu\left(r+r_{0}\right)\right]\right\}^{2 /(\delta-\lambda)} .
$$

In consequence the dimensionless background density parameter:

$$
\bar{\Omega}_{m}=\bar{\rho}_{m} / 3 \bar{H}^{2}=\frac{\bar{\rho}_{0, \gamma}}{3 A} \frac{\bar{a}^{\lambda(\lambda-\delta)}}{\bar{a}^{\lambda(\lambda-\delta)}+A / B},
$$

and,

$$
\bar{\Omega}_{\psi}=1-\bar{\Omega}_{m} .
$$

As before it is useful to have the magnitudes of observational interest written in terms of the redshift $z$. In the Jordan frame we have that $a(z)=a(0) /(z+1)$. We choose the normalization $a(0)=1 \rightarrow \bar{a}(0)=1$. Then, since $a=e^{-\psi /(2 \sqrt{\xi})} \bar{a}=\bar{a}^{n} \rightarrow \bar{a}=(z+1)^{-1 / n}$, where we have introduced the following definition for the constant $n$ : 


$$
n=\frac{2 \sqrt{\xi}-\lambda}{2 \sqrt{\xi}} .
$$

After these considerations the dimensionless density parameter and the Hubble expansion parameter can be written in the following way:

$$
\bar{\Omega}_{m}(z)=\frac{\bar{\rho}_{0, \gamma}}{3 A} \frac{(z+1)^{\left(\delta \lambda-\lambda^{2}\right) / n}}{(z+1)^{\left(\delta \lambda-\lambda^{2}\right) / n}+B / A},
$$

and,

$$
\bar{H}(z)=\sqrt{A} \sqrt{(z+1)^{\lambda \delta / n}+\frac{B}{A}(z+1)^{\lambda^{2} / n}},
$$

respectively. Other physical magnitudes of observational interest are the scalar field equation of state (and barotropic index):

$$
\bar{\omega}_{\psi}=-1+\bar{\gamma}_{\psi}, \quad \bar{\gamma}_{\psi}=\frac{\lambda^{2}}{3\left(1-\bar{\Omega}_{m}\right)},
$$

and the deceleration parameter: $\bar{q}=-\left(1+\dot{\bar{H}} / \bar{H}^{2}\right)$;

$$
\bar{q}=-1+\lambda^{2} / 2+(3 \gamma / 2) \bar{\Omega}_{m}
$$

\subsubsection{Relationship among frames}

Now it is useful to give the main physical magnitudes of observational interest also in the Jordan frame, as related with the EF magnitudes. Recall that $d t=e^{-\psi / 2 \sqrt{\xi}} d \bar{t}=\bar{a}^{-\lambda /(2 \sqrt{\xi})} d \bar{t}$, $a=e^{-\psi / 2 \sqrt{\xi}} \bar{a}=\bar{a}^{n}$ (see the definition for $n$ in Eq. (42)), and $\bar{T}_{a b}=e^{-\psi / \sqrt{\xi}} T_{a b} \rightarrow \rho_{m}=$ $e^{2 \psi / \sqrt{\xi}} \bar{\rho}_{m}=\bar{a}^{2 \lambda / \sqrt{\xi}} \bar{\rho}_{m}$. Other important relationships are the Hubble parameter:

$$
H=n e^{\psi /(2 \sqrt{\xi})} \bar{H}=n \bar{a}^{\lambda /(2 \sqrt{\xi})} \bar{H},
$$

the dimensionless density parameter,

$$
\Omega_{m}=\left(1 / n^{2}\right) \bar{\Omega}_{m},
$$

the dimensionless scalar field density parameter, ${ }^{8}$

$$
\Omega_{\phi}=1-\left(1 / n^{2}\right) \bar{\Omega}_{m},
$$

the scalar field equation of state (and the barotropic index):

\footnotetext{
${ }^{8}$ In the Jordan frame we define $\Omega_{i}=\rho_{i} / \rho_{\text {critical }}$, where $\rho_{\text {critical }}=3 e^{\phi} H^{2}$, so that the Friedmann constrain equation (26) can be written as: $1=\Omega_{m}+\Omega_{\phi}$.
} 


$$
\omega_{\phi}=-1+\gamma_{\phi}, \quad \gamma_{\phi}=\lambda \frac{(n / 3)(\lambda-1 / \sqrt{\xi})-\frac{1}{2}(\gamma / \sqrt{\xi}) \bar{\Omega}_{m}}{n^{2}-\bar{\Omega}_{m}},
$$

and the deceleration parameter,

$$
q=(1 / n)\left[\bar{q}+(1-n)\left(1-\lambda^{2} / 2\right)-(n / 2) \lambda / \sqrt{\xi}\right]
$$

This class of solutions depends (in principle) on 5 parameters: $\lambda, \xi, \bar{\rho}_{0}, \bar{V}_{0}$, and $\gamma$. The constants $A$ and $B$ are given in terms of the aforementioned parameters.

\subsection{The space of parameters}

In this subsection we will reduce the space of parameters by properly choosing the normalization for the Hubble parameter and by using some observational facts. In the Einstein frame the Friedmann constrain equation (29) means that $\bar{\Omega}_{m} \leq 1$. Besides, the solution $\bar{\Omega}_{m}=1$ is not stable so we are left with $\bar{\Omega}_{m}<1$. The same is true in the Jordan frame, where the Friedmann constrain equation (26) means that $0 \leq \Omega_{m} \leq 1$. On the one hand, $\Omega_{m}$ is a maximum at $z=\infty$ (the same is true for $\bar{\Omega}_{m}$ ). Then, we require that $\Omega_{m}(\infty)=1-\varepsilon$, where, as before, $\varepsilon$ is a small number. This, in turn, yields: $\bar{\Omega}_{m}(\infty)=n^{2}(1-\varepsilon)$ In consequence, $\bar{\rho}_{0, \gamma}=3 n^{2} A(1-\varepsilon)$. On the other hand, according to the observations, at present (in the JF) $\Omega_{m}(0)=\left(1 / n^{2}\right) \bar{\Omega}_{m}(0)=1 / 3$. This last equality implies that $\bar{\rho}_{0, \gamma}=n^{2}(A+B)$. Combining of the above constrains yields $B / A=2-3 \varepsilon$. In consequence, Eq. (43) and Eq. (44) can be written in the following way:

$$
\bar{\Omega}_{m}(z)=n^{2}(1-\varepsilon) \frac{(z+1)^{\left(\delta \lambda-\lambda^{2}\right) / n}}{(z+1)^{\left(\delta \lambda-\lambda^{2}\right) / n}+2-3 \varepsilon},
$$

and,

$$
\bar{H}(z)=\frac{\sqrt{\rho_{0, \gamma}}}{n} \sqrt{\frac{(z+1)^{\lambda \delta / n}+(2-3 \varepsilon)(z+1)^{\lambda^{2} / n}}{3(1-\varepsilon)}},
$$

respectively. The space of parameters $\left(\gamma, \lambda, \varepsilon, \bar{\rho}_{0, \gamma}\right)$ can be further reduced by choosing the normalization in which $H(0)=1 \Rightarrow \bar{H}(0)=1 / n \Rightarrow \bar{\rho}_{0, \gamma}=1$. In this case we are left with a three-parametric class of solutions spanned by the free parameters $(\gamma, \lambda, \varepsilon)$. In section 4 we will further constrain the free parameters $\lambda$ and $\varepsilon$ by means of comparison with supernovae observational data.

\subsection{Existence and Stability of the Solutions}

The stability of the system of dynamical equations (29-31) has been already studied in ([19]) for a single exponential of the kind $\bar{V}=\bar{V}_{0} e^{-g \psi}$. This study has been performed in the Einstein frame, however, the existence and stability of critical points already defined in the Einstein frame, remain unchanged under a conformal transformation back to the Jordan 
frame [19]. The system (29-31) can be arranged in the form of a plane-autonomous system if one introduces the variables:

$$
x=\frac{\dot{\psi}}{\sqrt{6} \bar{H}}, \quad y=\frac{\sqrt{\bar{V}}}{\sqrt{3} \bar{H}} .
$$

In this case one obtains [19]:

$$
y^{\prime}=-\sqrt{\frac{3}{2}} g x y+\frac{3}{2} y\left(2 x^{2}+\gamma\left(1-x^{2}-y^{2}\right)\right),
$$

and,

$$
x^{\prime}=-3 x+\sqrt{\frac{3}{2}} g y^{2}+\frac{3}{2} x\left(2 x^{2}+\gamma\left(1-x^{2}-y^{2}\right)\right)+W\left(1-x^{2}-y^{2}\right) .
$$

Following our previous result in section 2.C, that the linear relationship between the time derivative of the scalar field and the Hubble parameter leads to solutions being attractors of two possible kinds: scalar field dominated and scaling solutions, then one expects that the relationship (34) leads to the same result in the present situation.

The system described by (55) and (56) can have up to five fixed points depending on the values of $g, \gamma$ and $W$ [19]. However, when combined with the relationship (34), that leads to the fixed value: $x=\lambda / \sqrt{6}$, it leaves us with two possibilities (see the potential in Eq. (35)): 1) Either $g_{1}=\lambda \Rightarrow \bar{\Omega}_{\psi}=1$, and $\bar{\gamma}_{\psi}=\lambda^{2} / 3$, or 2) $g_{2}=\delta=3 \gamma / \lambda+\sqrt{2 / 3} W \Rightarrow \bar{\Omega}_{\psi}=$ $\lambda(\lambda-\sqrt{2 / 3} W) / 3 \gamma$, and $\bar{\gamma}_{\psi}=\gamma \lambda /(\lambda-\sqrt{2 / 3} W)$.

In the first case, in the Einstein frame, we have a scalar field dominated solution, meanwhile, in the second case we have a scaling solution. Solution 1) exists for $\lambda^{2}<6$ and is stable when $W-\sqrt{W^{2}+18 \gamma}<\sqrt{6} \lambda<W+\sqrt{W^{2}+18 \gamma}$. Meanwhile, solution 2) exists

for $\lambda>3 \gamma W /\left(\frac{9 \gamma(2-\gamma)}{2 \sqrt{6}}-1\right)$ and either $\lambda>-\frac{3 \sqrt{6} \gamma}{W+\sqrt{W^{2}+18 \gamma}}$ or $\lambda<-\frac{3 \sqrt{6} \gamma}{W-\sqrt{W^{2}+18 \gamma}}$. It is stable whenever it exists [19].

\section{Observational Testing}

Although we can not talk literally about observational testing of the solutions found (we point out that the observational facts are not yet conclusive in some cases and are not accurate enough in others), in this section we shall study whether our solutions agree with some observational constrains that are more or less well established. The main observational facts we consider are the following[2]:

1.- At present $(z=0)$ the expansion is accelerated $(q(0)<0)$.

2.- The accelerated expansion is a relatively recent phenomenon. Observations point to a decelerated phase of the cosmic evolution at redshift $z=1.7$. There is agreement in that transition from decelerated into accelerated expansion occurred at a $z \approx 0.5$ [24]. 
3.- The equation of state for the scalar field at present $\omega_{\phi}\left(\omega_{\varphi}\right) \sim-1$ (it behaves like a cosmological constant). With a $95 \%$ confidence limit $\omega<-0.6[25]$.

4.- Although, at present, both the scalar (quintessence) field and the ordinary matter have similar contributions in the energy content of the universe $\left(\Omega_{m}(0)=1 / 3 \Rightarrow \Omega_{\phi}(0)=2 / 3\right)$, in the past, the ordinary matter dominated the cosmic evolution, ${ }^{9}$ meanwhile, in the future, the quintessence field will dominate (it already dominates) and will, consequently, determine the destiny of the cosmic evolution.

Now we proceed to "observationally" test the solutions found in the cases studied in the former sections.

\subsection{Minimally coupled quintessence}

In the case of normal quintessence model studied in section 2, our solution depends on two parameters $\varepsilon$ and $k$ (we fix $\gamma=1$, meaning cold dark matter dominance at present). To constrain the values of these parameters we use the following facts:

1. Corasaniti and Copeland [26] found that the determination of the third peak in the BOOMERANG data limits the value today of equation of state $-0.93 \leq \omega_{\varphi}(0) \leq-1$

2. As stated before [27], nucleosynthesis predictions claims that at $95 \%$ confidence level. $\Omega_{\varphi}\left(1 M e V \simeq z=10^{10}\right) \leq 0.45$

3. During galaxy formation epoch [28], around $z \approx 2-4$, the value of quintessence density parameter is $\Omega_{\varphi}<0.5$

Using these constrains we bound the parameter space $(k, \varepsilon)$ by means of a computing code. These parameters range as $0 \leq \varepsilon \leq 0.045$ and $k>0$.

Now we select a pair from the above interval in order to compare our model with Supernova data. We first compare our model with the theoretical predictions of the Lambda Cold Dark Matter model $(\Lambda C D M)$ for modulus distance. Subsequently, we follow the procedure used in Ref. [29] to further test our model. However, this procedure, which implies direct comparison of our model with observations of effective apparent magnitudes of SNIa, can not be used to further constrain the space of parameters, since the free parameters in our model are not much sensible to these observations. For instance if we consider $\varepsilon=0.01$, the $\chi^{2}$ distribution has a minimum in $m_{0}$ direction ${ }^{10}$ at $m_{0}=23.97\left(\chi^{2}=62.5996\right.$ or $\chi^{2} /$ dof $=1.18$ per degree of freedom), however it has no minimum in the $k$ direction, meaning that the parameter $k$ can take any positive value. ${ }^{11}$ In fig. $1, \chi^{2}$ is plotted as function of the free parameters $k$ and $m_{0}$ (we chose $\varepsilon=0.01, k$ could be any value in the physically meaningful range). Although the model fulfils the observational requirements $1-4$ given at the beginning of this section, we point out that other observations should be considered in order to further constrain the space of parameters.

\footnotetext{
${ }^{9} \mathrm{~A}$ sufficiently long matter dominated decelerated phase is needed for the observed structure to develop from the density inhomogeneities [15]

${ }^{10} m_{0}$ is a parameter connected to the absolute magnitude and the Hubble parameter

${ }^{11}$ In the limit $k \rightarrow \infty$ the $\Lambda$ CDM model is recovered.
} 


\subsection{Brans-Dicke quintessence}

In this case our solution depends on two parameters $\lambda$ and $\varepsilon$ (recall that we fix $\gamma$ to be unity). We apply exactly the same procedure that in the former subsection to constrain the parameter space. However, in this case we should differentiate among the two relevant frames: The JF and the EF, in which Brans-Dicke theory can be formulated. We treat both formulations separately, bearing in mind that none of them could be taken to be the one with real physical meaning. We recall that, given the uncertain character of the dark energy (it dominates the energy content of the universe) and dark matter (it dominates the matter density in the universe), it could be possible that the dark energy and the dark matter, on the one side, and the ordinary (barionic) matter on the other, are minimally coupled with respect to different metrics (see Ref.[19] and references therein). Therefore, both conformal formulations are of physical significance.

When the aforementioned procedure is applied to the Einstein frame magnitudes of observational interest, one finds that the physically meaningful region in parameter space is bounded by $0 \leq \varepsilon \leq 0.045$ and $0<\lambda<0.37$. Meanwhile, in the Jordan frame one obtains that the physically meaningful range is $0 \leq \varepsilon \leq 0.045$ and $0.03<\lambda<0.38$.

It should be pointed out that, in all cases, the physically meaningful region in parameter space is chosen such that the main observational facts $1-4$ explained at the beginning of the section are fulfilled. As an illustration, in fig. 2, we show the evolution of both dimensionless background and scalar field energy densities \& $z$ for the Einstein frame magnitudes $\bar{\Omega}_{m}$ and $\bar{\Omega}_{\psi}$ respectively ${ }^{12}$ (the values of the free parameters are taken from the physically meaningful region in parameter space). In fig. 3, the evolution of the equation of state in both formulations $\bar{\omega}_{\psi}$ and $\omega_{\varphi} \& z$ is shown, meanwhile, in fig. 4, we plot the deceleration parameter in both frames $\bar{q}(z)$ and $q(z)$ to show the transition redshift when the evolution turns from decelerated into accelerated $(z *=0.54$ and $z *=0.56$ in the EF and in the JF respectively). It is seen that the differences in these magnitudes are not significant as one goes from one frame into the other. In fig. 5, as an illustration, we plotted the effective apparent magnitude observed [30] and calculated according to our model with parameters $(\varepsilon=0.01, \lambda=0.2)$ to show the good fitting (the results are within $1 \sigma$ expectation).

We want to stress that, as in the former case (subsection 4.1), the free parameters of the model are not much sensible to the SNIa data so, the standard procedure used for instance in Ref. [29], is not suitable for further constraining of the space of parameters of the model. Other observational evidence could be considered for this purpose.

\section{Conclusions}

We have found a new parametric class of exact cosmological scaling solutions in gravity theory minimally coupled to a self-interacting scalar field and in Brans-Dicke theory with a self-interacting BD scalar, by assuming a linear relationship between the Hubble expansion

\footnotetext{
${ }^{12}$ The Jordan frame magnitudes $\Omega_{m}$ and $\Omega_{\varphi}$ do not differ significantly from the corresponding EF ones.
} 
parameter and the time derivative of the scalar field. This relationship is suggested by an implicit symmetry of the field equations.

We point out that it is not necessary to make any a priori assumptions about the functional form of the self-interaction potential or about the scale factor behavior. These are obtained as outputs of the assumed relationship once one integrates the field equations explicitly. As a result a peculiar class of potentials arise: a combination of exponentials with some dependence on the barotropic index of the ordinary matter fluid. This kind of potential is of interest for models of dark energy. Recall that a single exponential potential can lead to one of the following scaling attractor solutions[12]: 1) a late time attractor where the scalar field mimics the evolution of the background barotropic fluid, and 2) the late time attractor is the scalar field dominated solution. In consequence, a combination of exponentials should allow for a scenario in which the universe evolves through a radiation-matter regime (attractor 1) and at some recent epoch evolves into the scalar field dominated regime (attractor 2).

By inspection of the stability and existence of the solutions found, we show that the assumed linear relationship between the Hubble parameter and the time derivative of the scalar field can lead always (both in standard minimally coupled and in Brans-Dicke models of dark energy) to attractor scaling solutions. Otherwise, the assumed relationship acts as a selection principle: only two of five critical points in phase space agree with it.

In all cases, the relevance of the solutions found is outlined and the space of parameters is constrained with the help of a computed code that considers some well established observational facts. The models explored here are tested with the SNIa observational data. Agreement with observations is always within $1 \sigma$ expectation. It is pointed out, however, that the procedure of Ref. [29], can not be used to further constrain the parameter space, since the free parameters in the models of interest are not sensible to the luminosity distance SNIa observational data. Nevertheless, the scaling solutions found in both cases, meet the main observational facts considered in this paper with acceptable accuracy (points 1-4 at the beginning of section 4.). It is also pointed out that other observational data should be considered to further constrain the space of parameters.

In the case we deal with minimally coupled quintessence, the model explored in this paper is a particular case of the one in Ref.[12]. However, in that paper the authors did not consider any exact solutions at all but their aim was to numerically explore the model. In the Brans-Dicke case, as far as we know, the combination of exponentials has not been explored yet, although the stability and existence of scaling solutions in Brans-Dicke quintessence with a single exponential potential has been studied, for instance, in Ref.[19].

We thank our colleagues Rolando Cardenas, Diosdado Villegas and Carlos R. Fadragas for helpful comments and Indrajit Chakrabarty for pointing to us Ref.[19]. We acknowledge the MES of Cuba by financial support of this research. 


\section{References}

[1] S. Perlmutter et al., Astrophys. J. 517 (1999) 565-586, astro-ph/9812133; A. G. Riess et al., Astron. J. 116 (1998) 1009-1038, astro-ph/9805201; Astrophys.J. 560 (2001) 49-71, astro-ph/0104455.

[2] M. S. Turner, astro-ph/0202008 (To appear in the Proceedings of 2001: A Spacetime Odyssey (U. Michigan, May 2001, World Scientific)).

[3] V. Faraoni, Int. J. Mod. Phys. D11 (2002) 471-482, astro-ph/0110067; E. Gunzig, A. Saa, L. Brenig, V. Faraoni, T.M. Rocha Filho and A. Figueiredo, Phys. Rev. D63 (2001) 067301, gr-qc/0012085.

[4] A. Riazuelo, J.-P. Uzan, Phys. Rev. D66 (2002) 023525, astro-ph/0107386; J.-P. Uzan, Phys. Rev. D59 (1999) 123510, gr-qc/9903004; F. Perrotta, C. Baccigalupi, astroph/0205245, Phys. Rev. D65 (2002) 123505, astro-ph/0201335; F. Perrotta, C. Baccigalupi, S. Matarrese, Phys. Rev. D61 (2000) 023507, astro-ph/9906066; T. Chiba, Phys. Rev. D64 (2001) 103503, astro-ph/0106550.

[5] D. F. Torres, Phys. Rev. D66 (2002) 043522, astro-ph/0204504.

[6] P. J. E. Peebles and Bharat Ratra, astro-ph/0207347.

[7] B. Ratra and P. J. E. Peebles, Phys. Rev. D37 (1988) 3406.

[8] L. P. Chimento and A. S. Jakubi, Int. J. Mod. Phys. D5 (1996) 71-84, gr-qc/9506015.

[9] A. A. Starobinsky, Grav. Cosmol. 4 (1998) 88-99, astro-ph/9811360.

[10] C. Rubano and P. Scudellaro, Gen. Rel. Grav. 34 (2002) 307-328, astro-ph/0103335.

[11] L. A. Urena-Lopez, T. Matos, Phys. Rev. D62 (2000) 081302, astro-ph/0003364.

[12] T. Barreiro, E. J. Copeland and N. J. Nunes, Phys. Rev. D61 (2000) 127301, astroph/9910214; E. J. Copeland, N. J. Nunes, F. Rosati, Phys. Rev. D62 (2000) 123503, hep-ph/0005222.

[13] G. F. R. Ellis and M. Madsen, Class. Quant. Grav. 8 (1991) 667.

[14] C. Uggla, R. T. Jantzen and K. Rosquist, Gen. Rel. Grav. 25 (1993) 409.

[15] A. A. Sen, S. Sethi, Phys. Lett. B532 (2002) 159-165, gr-qc/0111082.

[16] T. D. Saini, S. Raychaudhury, V. Sahni and A. A. Starobinsky, Phys. Rev. Lett. 85 (2000) 1162-1165, astro-ph/9910231.

[17] Y. Gong, Class. Quant. Grav. 19 (2002) 4537-4542, gr-qc/0203007. 
[18] C. Rubano and J. D. Barrow, Phys. Rev. D64 (2001) 127301, gr-qc/0105037.

[19] D. J. Holden and D. Wands, Phys. Rev., D61, 043506 (2000), gr-qc/9908026.

[20] L. P. Chimento, Class. Quantum Grav. 15 (1998) 965-974; J. M. Aguirregabiria and L. P. Chimento, Class. Quantum Grav. 13 (1996) 3197-3209; L. P. Chimento , A. E. Cossarini and N. A. Zuccala; Class. Quantum Grav. 15 (1998) 57-74; L. P. Chimento, N. A. Zuccala and V. Mendez; Class. Quantum Grav. 16 (1999) 3749-3763.

[21] O. Arias, R. Cardenas, I. Quiros, Nucl. Phys. B643 (2002) 187-200, hep-th/0202130.

[22] E. J. Copeland, A. R. Liddle and D. Wands, gr-qc/9711068.

[23] C. M. Will, Theory and Experiment in Gravitational Physics (Cambridge University Press, 1993); gr-qc/0103036.

[24] A. Riess, astro-ph/0104455; M. S. Turner and A. Ries, astro-ph/0106051 (Astrophys. J., in press).

[25] S. Perlmutter, M. S. Turner and M. White, Phys. Rev. Lett. 83 (1999) 670-673, astroph/9901052; M. S. Turner and M. White, Phys. Rev. D56 (1997) R4439.

[26] P. S. Corasaniti and E. J. Copeland, Phys. Rev. D65 (2002) 043004, astro-ph/0107378.

[27] R. Bean, S. H. Hansen, and A. Melchiorri, Nucl. Phys. Proc. Suppl. 110 (2002) 167 , astro-ph/0201127.

[28] M. S. Turner, Nucl. Phys. Proc. Suppl. 72 (1999) 69, astro-ph/9811366.

[29] M. Pavlov, C. Rubano, M. Sazhin and P. Scudellaro, Astrophys.J. 566 (2002) 619, astro-ph/0106068.

[30] S. Perlmutter, G. Aldering, G. Goldhaber et al., ApJ 517 (1999) 565. 
Figure 1: The function $\chi^{2}$ is plotted as function of the free parameters $k$ and $m_{0}$ (we chose $\varepsilon=0.01$. As seen $k$ could be any value in the physically meaningful range so, SNIa luminosity observations do not allow for further constrain of the parameter space. Other observations could be considered for this purpose.

Figure 2: We show the evolution of both dimensionless background and scalar field energy densities $\& z$ for the Einstein frame magnitudes $\bar{\Omega}_{m}$ and $\bar{\Omega}_{\psi}$. The parameters $\lambda=0.2$ and $\varepsilon=0.01$ have been chosen so that the SNIa data is reproduced within $1 \sigma$. Equality of matter and quintessence energy density occurs approximately at $z \approx 0.3-0.4$.

Figure 3: The evolution of the equation of state in both formulations $\bar{\omega}_{\psi}$ and $\omega_{\varphi} \& z$ is shown. The equation of state for the quintessence field in the Einstein frame is never positive.

Figure 4: We plot the deceleration parameter in both frames $\bar{q}(z)$ and $q(z)$ to show the transition redshift when the evolution turns from decelerated into accelerated: $z *=0.54-$ 0.56. It is seen that the differences in these magnitudes are not significant as one goes from one frame into the other.

Figure 5: As an illustration, we plotted the effective apparent magnitude observed [30] and calculated according to our model with parameters $(\varepsilon=0.01, \lambda=0.2)$ to show the good fitting. The results are within $1 \sigma$ expectation. 


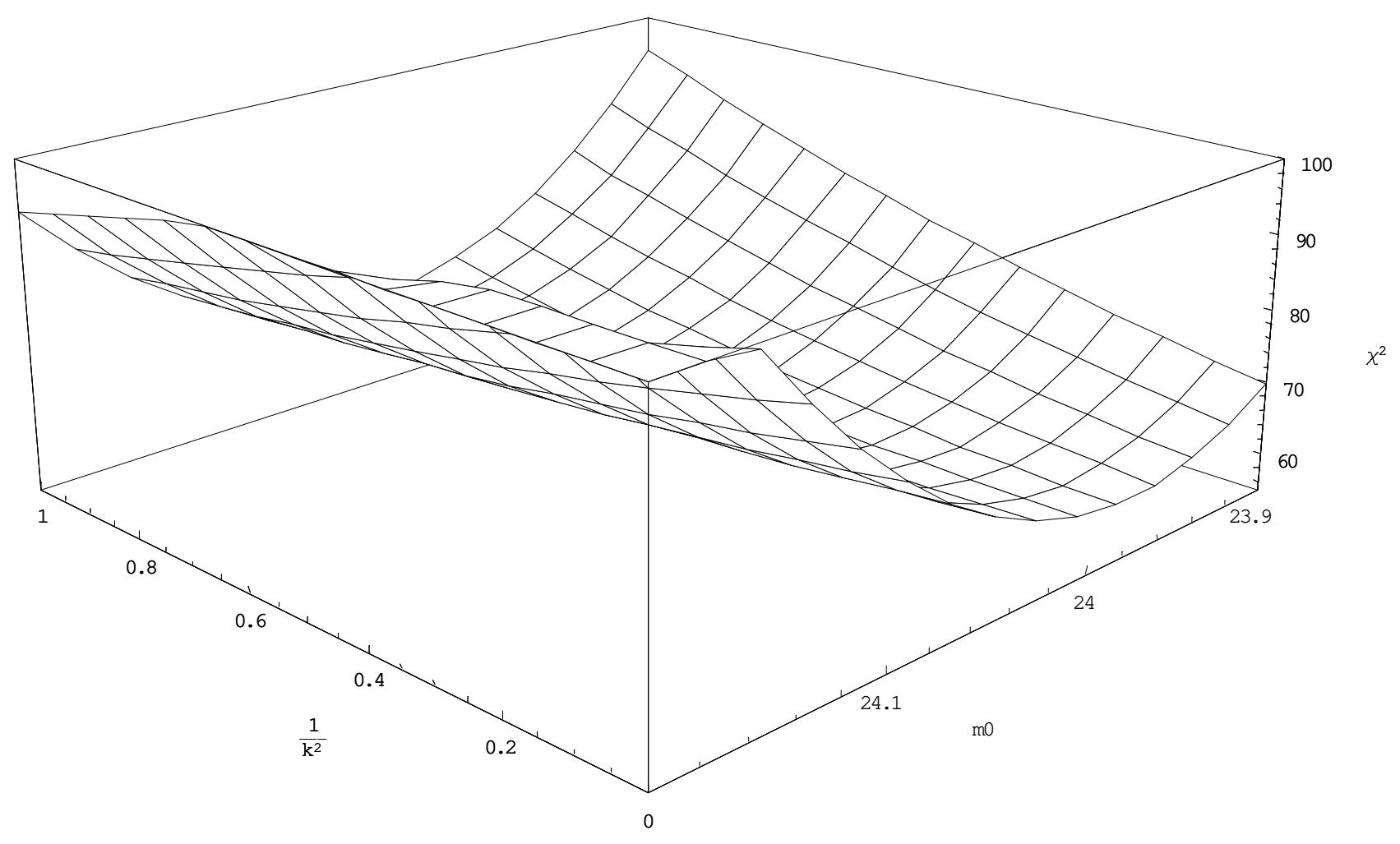




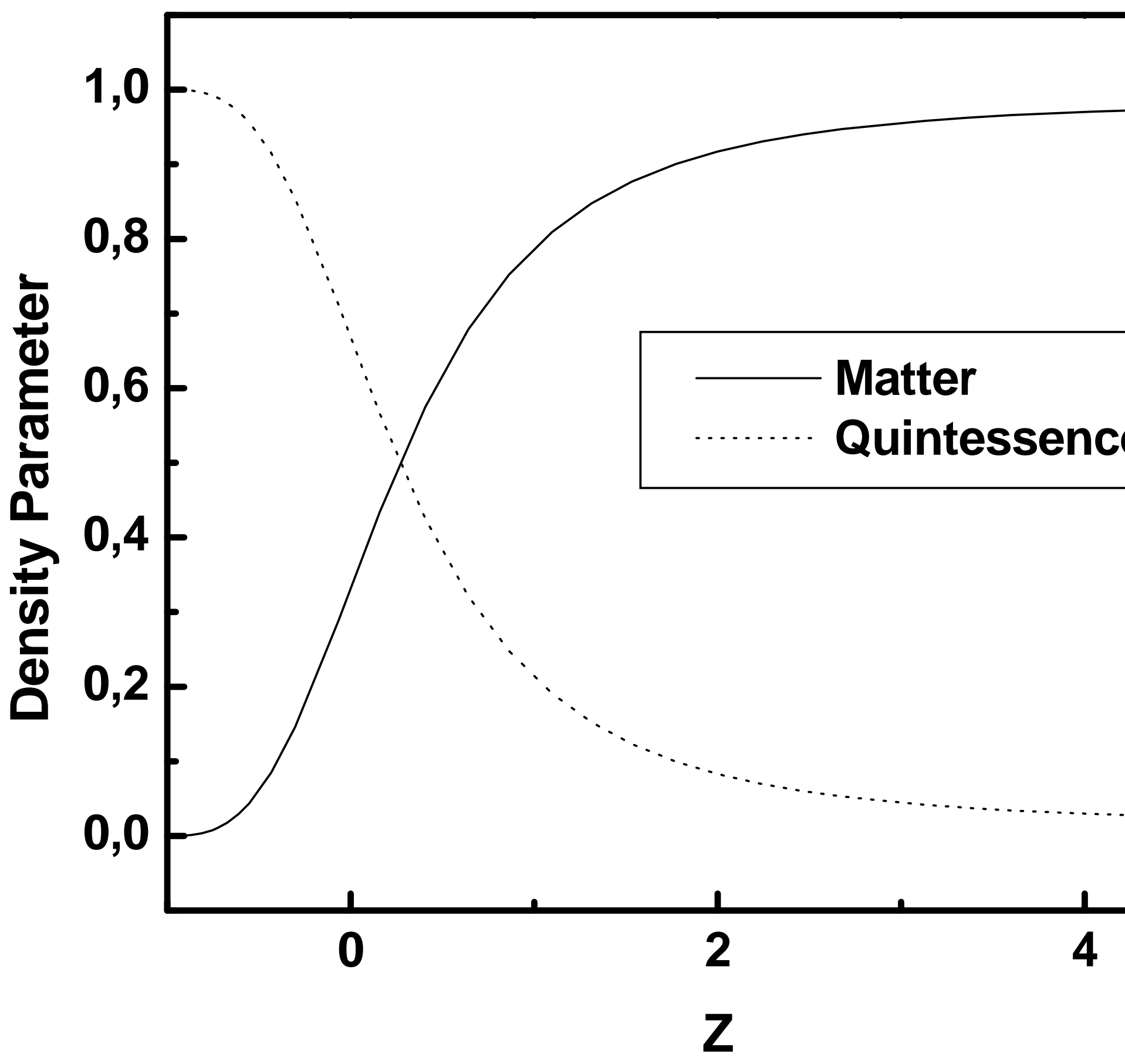




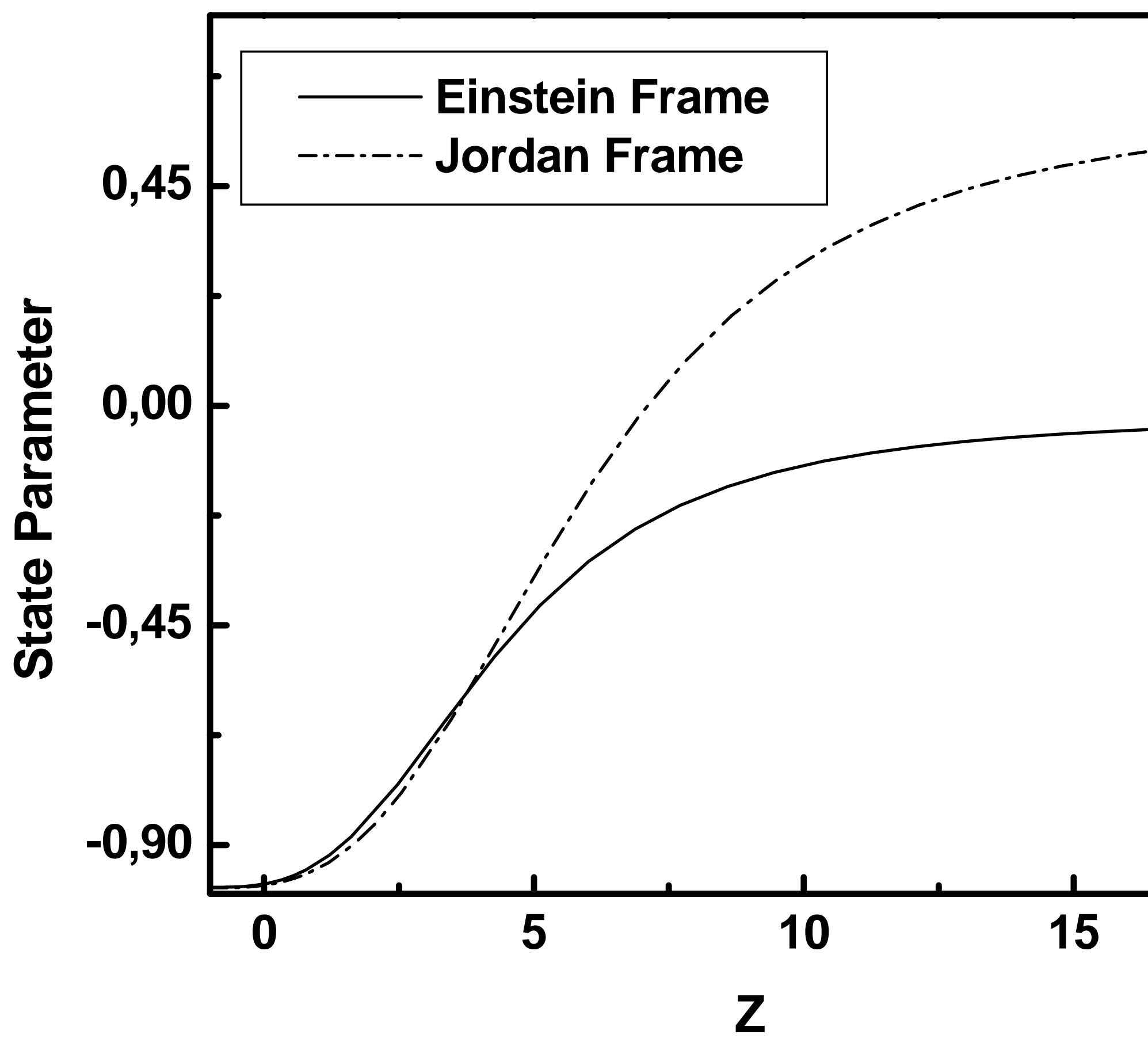




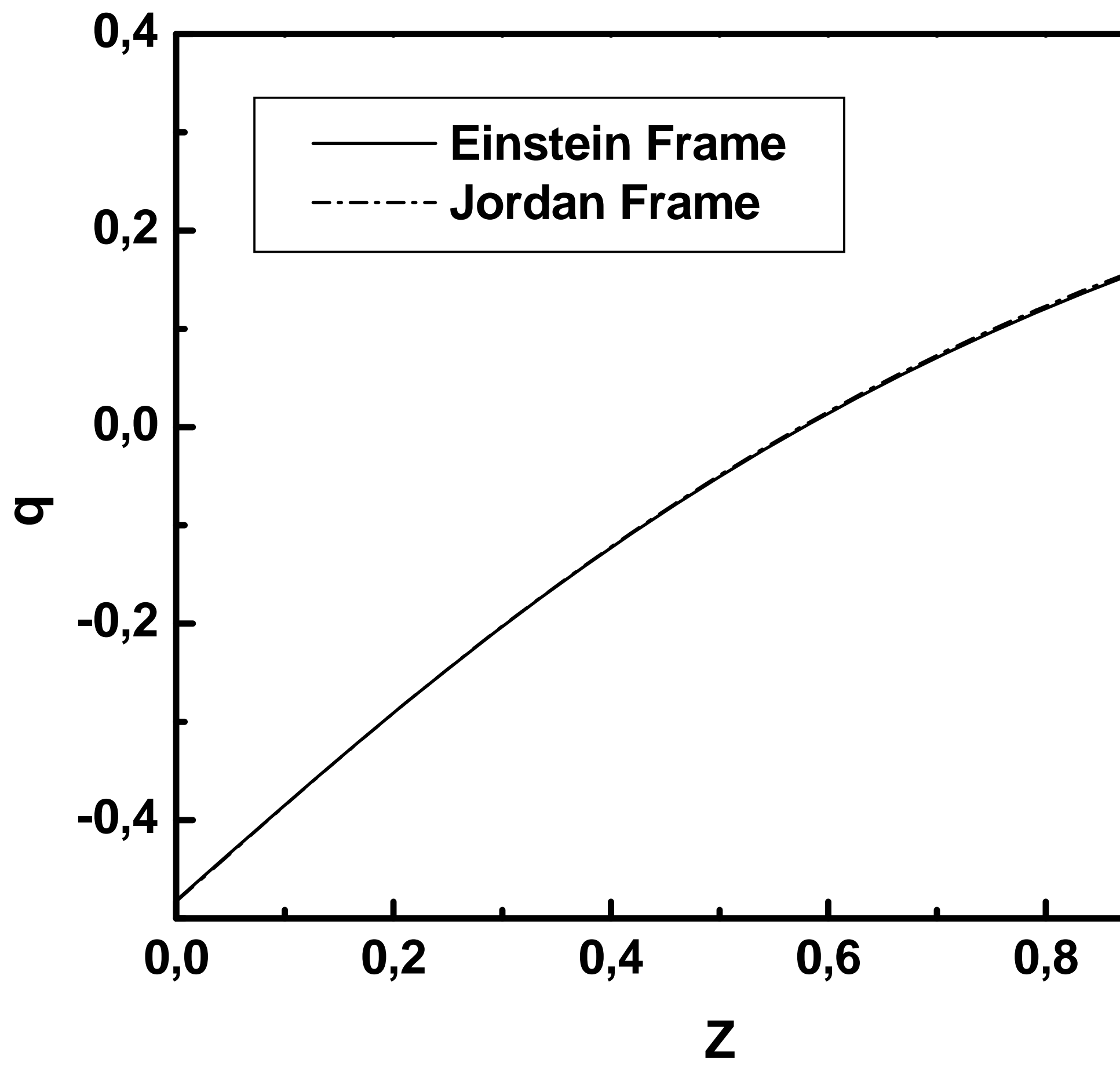




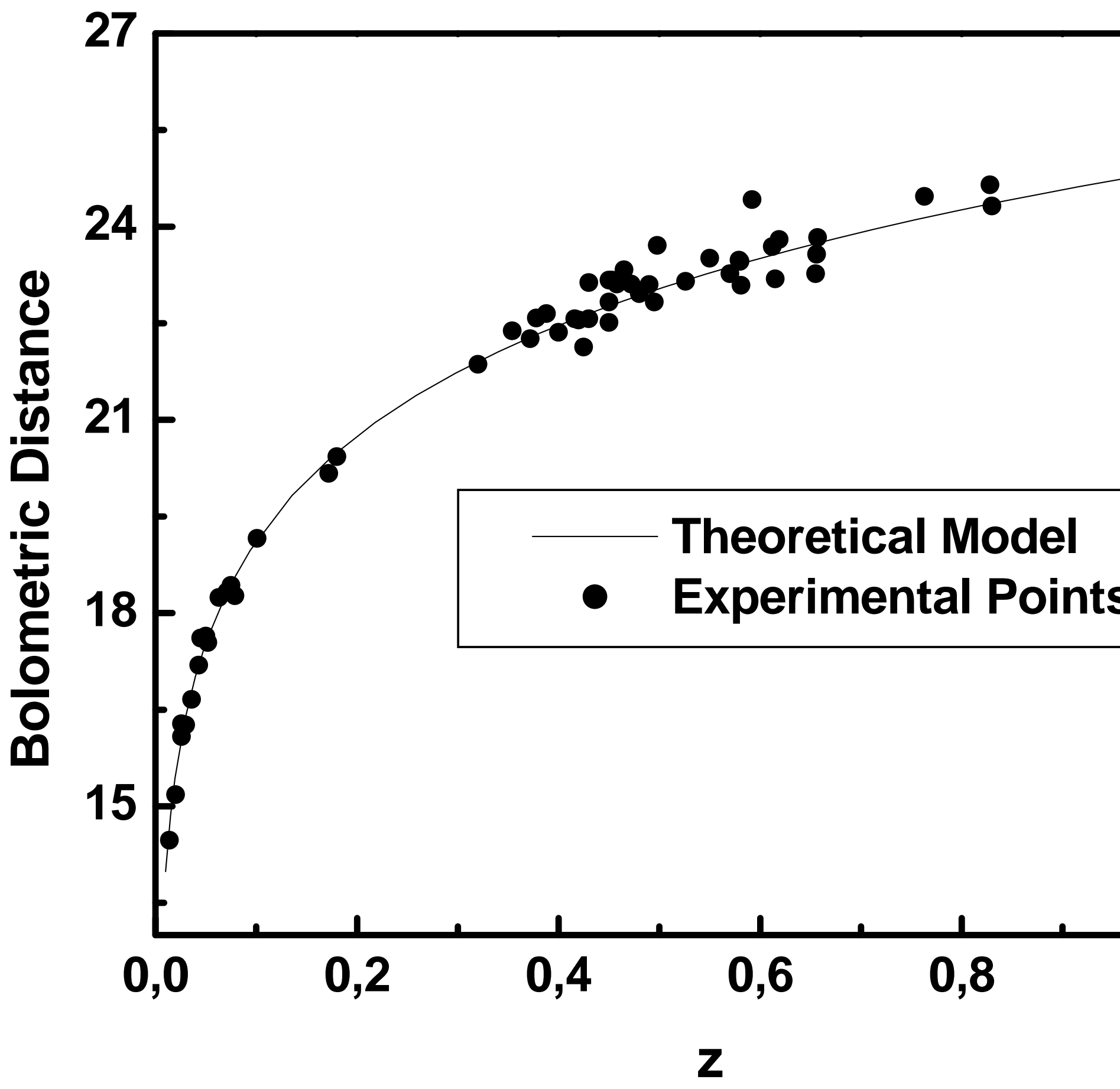

